\title{
Automated Crystallographic Identification of Atom Probe's Ion Desorption Map
}

\author{
Yimeng Chen ${ }^{1}$, Katherine P. Rice ${ }^{1}$, Ty J. Prosa ${ }^{1}$, David A. Reinhard ${ }^{1}$, Brian P. Geiser ${ }^{1}$, Matt M. Nowell ${ }^{2}$ \\ and Stuart I. Wright ${ }^{2}$ \\ 1. CAMECA Instruments, Inc., Madison, WI, USA \\ 2. EDAX, Draper, UT, USA
}

Atom probe tomography can provide high accuracy analysis for local composition at the atomic-scale, but structural analysis often relies on alternative techniques that are carried out separately. However, atom probe results often already contain pieces of crystallographic information which can be used in identifying crystal structure, determining crystallographic orientation, and measuring lattice parameters of matrix and precipitate phases. It has similarities to conventional work using field ion microscopy, but rather than ionizing imaging gases, atom probe uses field evaporated ions for 3D chemical imaging.

In crystalline materials, especially for metals, trajectory aberrations result in lower ion hit intensity at poles and zone lines, providing unique field desorption maps similar to crystal stereographic projection maps. Accuracy in APT reconstruction is an important aspect but time consuming and not always easy to achieve [1]. Reconstruction based on lattice spacing is one of the more reliable methods to improve reconstruction accuracy [2-3]. Post-reconstruction analysis such as the 3D Hough transformation has been reported for pole indexing [4]. In order to facilitate APT reconstructions using lattice spacing for known structure materials, we will demonstrate automated pole indexing using a customized OIM ${ }^{\mathrm{TM}}$ for IVAS $^{\mathrm{TM}}$ software package from $\operatorname{EDAX}^{\circledR}$ [5]. Here, the same algorithms used to index electron backscatter diffraction (EBSD) patterns are applied to atom probe field desorption maps.

There are strong similarities between field desorption maps and EBSD patterns. Both show poles and zone lines of crystallographic projection maps. However, the mechanisms that create the patterns are different. EBSD patterns follow electron diffraction rules but field desorption maps are not bound by same these rules. For example, forbidden reflectors in electron diffraction can appear in the field desorption maps. A customized structure database is necessary to index field desorption maps accurately while relying on the same Hough transform.

Patterns of well-ordered high purity materials are generally good and can be indexed automatically. Figure 1a and $\mathrm{b}$ show examples of high quality field desorption maps from BCC tungsten and FCC aluminum, respectively. In the output, the software provides several index solutions from which the user may choose. A confidence of indexing parameter and mismatch of fit are important parameters to examine in justifying the best choice for indexing. Figure 1c and $\mathrm{d}$ are maps after image processing superimposed with an index overlay. The best solution is the one with the highest index confidence and the lowest degree of mismatch.

With the ability to index all the poles in a field desorption map, the reconstruction parameters can be adjusted to have optimized d-spacing values for all the poles. Figure $2 \mathrm{a}$ is a $5 \mathrm{~nm}$ slice of a tungsten atom map cutting through (114), (116) and (002) poles. Lattice planes are clearly visible on low index poles with the correct $\mathrm{d}$-spacing values. The insets in Figure $2 \mathrm{c}$ and $\mathrm{d}$ are lateral spatial distribution maps (SDMs) [6] for (002) and (114) poles showing the regularity of neighboring atoms in the x-y plane. 
In this work, indexing field desorption maps with OIM software for IVAS will be demonstrated. The use of dedicated structure files will be discussed. Examples of indexing poor contrast field desorption maps from highly alloyed materials will also be exhibited.

\section{References:}

[1] T.J Wilkes et al, Metallography 7.5 (1974), p. 403.

[2] P. Bas et al, Appl. Surfa. Sci. 87-8 (1995), p. 298.

[3] B. Gault et al, in “Atom Probe Microscopy” (Springer New York, 2012) p. 290

[4] L. Yao et al, Ultramicroscopy 111 (2011), p. 458.

[5] B. L. Adams, S. I. Wright, and K. Kunze, Metallurgical Transactions A 24(4) (1993), p. 819.

[6] B. P Geiser et al, Microscopy and Microanalysis 13 (2007) p. 437.
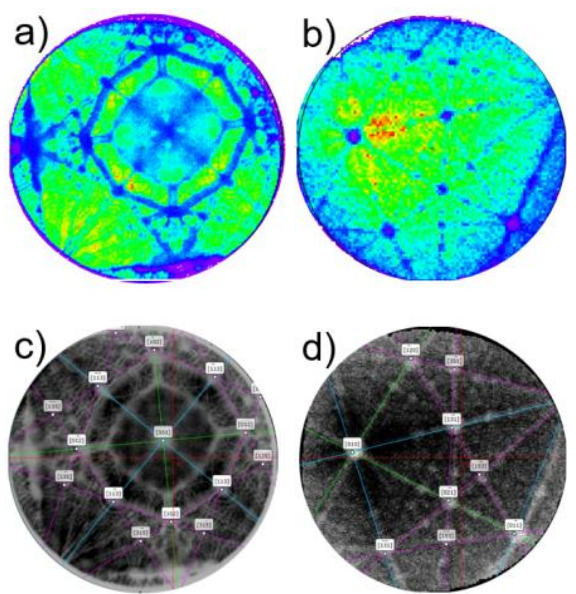

Figure 1. Field disorption maps of a) tungsten and b) aluminum. c) and d) are the same maps after imaging processing. The solution of index was chosen for the highest index confidence and the lowest degree of mismatch.

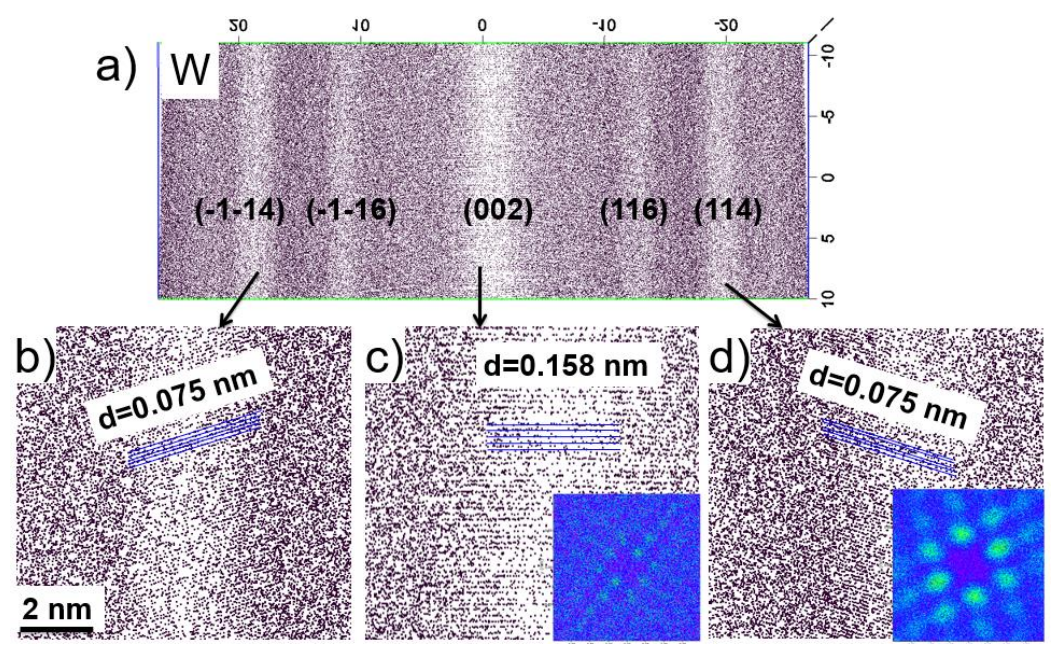

Figure 2. a) is a $5 \mathrm{~nm}$ slice of atom maps of tungsten aligned to its (002) pole. The reconstruction parameters were adjusted to have correct d-spacing values for the (114) and (002) poles. The inserts are lateral spatial distribution maps of neighboring atoms in these poles. 